\title{
On the Motion of a Vortex in a Non-Uniform Pressure Field
}

(Provisional Report)

\author{
by
}

S.Syöno

Geophysical Institute, Tokyo University and Meteorological Research Instituts

(Received June 5, 1951)

\begin{abstract}
In this paper, equations of motion of a vortex in a non-uniform pressure field are obtained. Integrating the equations of motion over the domain of a vortex and separating the motion into that of a vortex as a whole and that within the vortex, equations of motion. of a vortex are obtained. Among other things, important results obtained are as follows: (1) By the effect of the Rossby's force and the Coriolis force, the vortex moves on a cycioidal path, a cyclonic vortex eastward and an anticyclonic vortex westward. (2) The motion of a vortex in a uniform pressure gradient field is composed of four types of motion. (3) The well-known fact that cyclones tend to isallobaric lows is explained.
\end{abstract}

\section{Introduetion}

The forecasting of the motion of tropical cyclones is one of the most important problems from the practical point of view. The principles used for this purpose are steering of the upper current and the attraction to the isallobaric low. However, we had no satisfactory theory on the motion of a vortex as a who'e. In 1944, Professors Bjerknes and Holmboe put forward a theory on the motion of a vortex based on the displacement of pressure patterns. In 1948 and 1949, Prof. Rossby treate the $^{-}$ motion of a vortex and obtained a new force caused by the meridional change of the Coriolis force. As was remarked by Dr. Kuo (1950), the Rossby's force cannot explain the southward motion of upper cut-off lows. Further the Rossby's theory does not contain the inertial oscillation due to the Corjolis force. In 1950, Drs, Yen and Kuo treated the same problem under the assumption that the motion is irrotational or the vorticity is uniform. In the present paper, equations of motion of a vortex in a non-uniform prssure field are obtained. Some simplified excmples are treated, by which the meandering motion of tropical cyclones may be explained. Finally, since the equations contain the pressure gradient force, by certain operation, the isallobaric velocity of the vortex is obtained besides the geostrophic velocity.

\section{Preliminary Analysis}

We consider a unit slice of the vortex. Let the latitude and the longitude of

Division of Meteorology, Geophysical Institute, Tokyo University, Contribution No. 37 


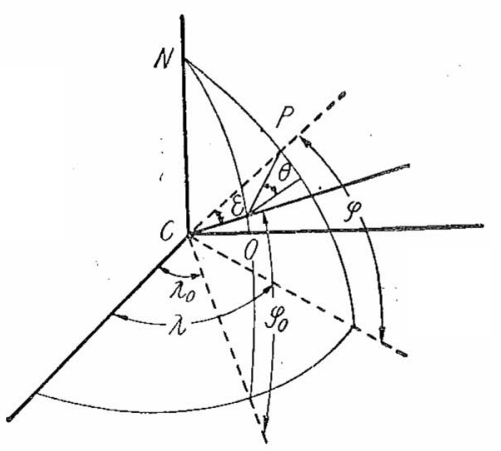

Fig. 1

the center of the vortex $O$ be $\lambda_{0}$ and $\varphi_{0}$, the longitude and the latitude of a parcel within the vortex be $\lambda$ and $\varphi$. We consider local cylindrical coordinates referred to $O$. The coordinates of $P$ referred to $O$ is $r$ and $\theta$, where $r$ and $\theta$ denote the radial distance and the azimuthal angle of $O P$ from the eastward zonal axis taken in anticyclonic sense.

We consider a spherical triangle NOP. If $r$ is small compared with the radius of the earth $R$, the arc of a great circle passing through $O$ and $P$ is substituted by a straight line element, $O P$ and the angle between the great circle and the zonal circle is substituted by $\theta$. (Fig. 1 ) From the formula of spherical trigonometry, we get

$$
\cos \left(\frac{\pi}{2}-\varphi\right)=\cos \left(\frac{\pi}{2}-\varphi_{0}\right) \cos \varepsilon+\sin \left(\frac{\pi}{2}-\varphi_{0}\right) \sin \varepsilon \cos \left(\frac{\pi}{2}-\theta\right),
$$

where $\varepsilon$ is $\angle P C O$ and is approximately equal to $r / R$. Expanding this equation with respect to $r / R$, we get

$$
\sin \varphi=\sin \varphi_{0}+\cos \varphi_{0} \frac{r}{R} \sin \theta+O\left(\left(\frac{r}{R}\right)^{2}\right)
$$

\section{Equations of motion of a vortex as a whole}

Neglecting the vertical motion and the viscosity, the equations of motion are

$$
\begin{aligned}
& \rho\left(\frac{d v_{x}}{d t}-2 \omega \sin \varphi v_{y}\right)=-\frac{\partial P}{\partial x}, \\
& \rho\left(\frac{d v_{y}}{d t}+2 \omega \sin \varphi v_{x}\right)=-\frac{\partial P}{\partial y},
\end{aligned}
$$

where $x$ and $y$ are coordinates taken positive eastward and northward, $v_{x}$ and $v_{y}$ are $x$ - and $y$-components of velocity. The density $\rho$ is assumed constant. $P$ and $\omega$ are the pressure, the angular velocity of the earth rotation. Now we put

$$
v_{x}=V_{x}+v_{x}{ }^{\prime}, \quad v_{y}=V_{y}+v_{y^{\prime}},
$$

where $V_{x}$ and $V_{y}$ are defined by

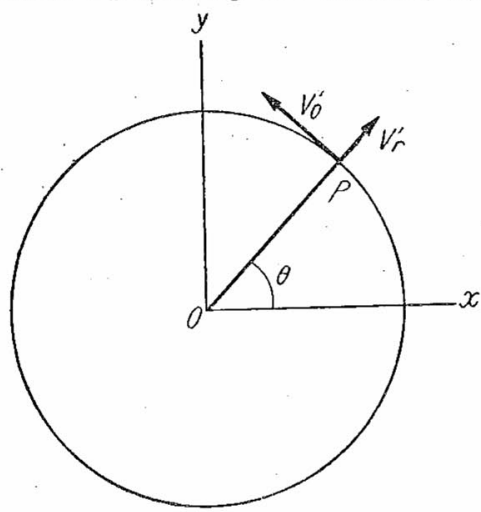

Fgi. 2
(6) $\quad V_{x}=\frac{1}{S} \iint_{S} v_{x} d \mathrm{~S}, \quad V_{y}=\frac{1}{S} \iint_{S} v_{y} d \mathrm{~S}$.

Here $S$ denotes the area of the unit slice of vortex, then $V_{x}$ and $V_{y}$ mean the velocity components of the vortex as a whole. $v_{x}^{\prime}$ and $v_{y}^{\prime}$ are velocity components of the motion referred to the centre of the vortex and represented with $v_{r^{\prime}}{ }^{\prime}$ and $v_{\theta}{ }^{\prime}$, where $v_{r}^{\prime}$ and $v_{\theta}{ }^{\prime}$ are $r-$ and $\theta-$ components of the velocity. Using cylindrical coordinates, we get

$$
\begin{aligned}
& \text { (7) } \quad v_{x^{\prime}}=v_{x^{\prime}}{ }^{\prime} \cos \theta-v_{\theta}{ }^{\prime} \sin \theta, \\
& \text { (8) } \quad v_{y^{\prime}}=v^{\prime}{ }^{\prime} \sin \theta+v_{\theta}{ }^{\prime} \cos \theta
\end{aligned}
$$

Generally $v_{r}^{\prime}$ and $v_{\theta}^{\prime}$ are expressed with Fourier 
series of $\theta$ with coefficients, which are functions of $r$. From the definitions of $V_{x}$ and $V_{y}$, the integrals of $v_{x}^{\prime}$ and $v^{\prime} y^{\prime}$ vanish. Using these conditons, we get

$$
\begin{aligned}
& v_{r}{ }^{\prime}=a_{0}(r)+\sum_{n=2}^{\infty} a_{n}(r) \cos n \theta+\sum_{n=2}^{\infty} \widetilde{a_{22}}(r) \sin n \theta, \\
& v_{\theta}{ }^{\prime}=b_{0}(r)+\sum_{n=2}^{\infty} b_{n}(r) \cos n \theta+\sum_{n=2}^{\infty} \widetilde{b_{n 2}}(r) \sin n \theta .
\end{aligned}
$$

Because the pattern of the motion of higher order than the second is too much complicated, we take only the first terms, i.e. the vortex is assumed to be symmetric. We put $\mathrm{P}=\bar{P}+p^{\prime}$, where $\bar{P}$ gives the general pressure field of the large scale compared with the scale of the vortex, and $p^{\prime}$ gives the pressure fjeld of the small scale accompanied by the vortex.

Using the condition of the conservation of mass $\frac{d}{d t} \iint_{S} d S=0$, the operators $\frac{d}{d t}$ and $\iint{ }_{s}$ may be commuted, so that we get

$$
\iint_{S} \frac{d v_{x}}{d t} d S=\frac{d}{d t} \iint_{S} v_{x} d S=S \frac{d V_{x}}{d t}
$$

Similarly

$$
\iint_{S} \frac{d v_{y}}{d t}=S \frac{d V y}{d t}
$$

Introducting (5) into (3) and (4) and integrating and using (2), (6), (7), (8), (9), (10), (11) and (12), we get

$$
\begin{aligned}
& \frac{d V_{x}}{d t}-2 \omega \sin \varphi_{0} V_{y}=-\frac{1}{\rho} \frac{\partial \bar{P}}{\partial x}+G_{x}, \\
& \frac{d V_{y}}{d t}+2 \omega \sin \varphi_{0} V_{x}=-\frac{1}{\rho} \frac{\partial \bar{P}}{\partial y}+G_{y},
\end{aligned}
$$

where

$$
\begin{aligned}
G_{x} & =\frac{2 \omega}{S} \iint_{S} \sin \varphi v_{y}^{\prime} d S-\frac{1}{\rho S} \iint_{S} \frac{\partial p^{\prime}}{\partial x} d S, \\
G_{y} & =\frac{2 \omega}{S} \iint_{S} \sin \varphi v_{x}^{\prime} d S-\frac{1}{\rho S} \iint_{S} \frac{\partial p^{\prime}}{\partial y} d S,
\end{aligned}
$$

and the bars denote the mean values within the vortex. Now we assume

$$
\begin{aligned}
& v_{r^{\prime}}=a_{0}(r), \\
& v_{\theta}{ }^{\prime}=b_{0}(r), \\
& p^{\prime}=c_{0}(r)+\sum_{n=1}^{\infty} c_{3 z}(r) \cos n \theta+\sum_{n=1}^{\infty} \widetilde{c_{n n}}(r) \sin n \theta .
\end{aligned}
$$

Introducting (17), (18) and (19) into (15) and (15) and taking account of (2), (7) and (8), we get

$$
\begin{aligned}
& G_{x}=\frac{2 \omega}{a^{2} R} \cos \varphi_{0} \int_{0}^{a} a_{0}(r) r^{2} d r-\frac{1}{\rho a} c_{1}(a), \\
& G_{y}=\frac{2 \omega}{a^{2} R} \cos \varphi_{0} \int_{0}^{a} b_{0}(r) r^{2} d r-\frac{1}{\rho x} \widetilde{c_{1}}\left(a_{u}\right),
\end{aligned}
$$


where $a$ is the radius of the vortex. In the above equations, it is to be noticed that even if we take the general form (19), the first order only is effective and if $c_{1}(a)=0, \widetilde{c_{1}}(a)=0$, the pressure gradiant force vanishes.

Then the eqnations of motion of a circular vortex become as follows;

$$
\begin{aligned}
& \frac{d V_{x}}{d t}-2 \omega \sin \varphi_{0} V_{y}=-\frac{1}{\rho} \frac{\partial \bar{P}}{\partial x}-\frac{1}{\rho c} c_{1}(a)+\frac{2 \omega}{a^{2} R} \cos \varphi_{0} \int_{0}^{a} a_{0}(r) r^{2} d r, \\
& \frac{d V_{y}}{d t}+2 \omega \sin \varphi_{0} V_{x}=-\frac{1}{\rho} \frac{\partial \bar{P}}{\partial y}-\frac{1}{\rho a} \widetilde{c_{1}}(a)+\frac{2 \omega}{a^{2} R} \cos \varphi_{0} \int_{0}^{a} b_{0}(r) r^{2} d r .
\end{aligned}
$$

The third term on the right-hand side of (22) means a force directed westward for the converging centre which was suggested by Rossby (1948). The third term on the right-hand side of (23) is the Rossby's force.

\section{Special examples}

(i) Motion of a circular vortex in a uniform pressure field.

Hereafter we shall drop the subscripts 0 and use them in another meaning.

When a circular vortex of radius $a$ is rotating with an angular vetocity $\Omega$ in a uniform pressure field, the equations of motion become

$$
\begin{aligned}
& \frac{d V_{x}}{d t}-2 \omega \sin \varphi V_{y}=0, \\
& \frac{d V_{y}}{d t}+2 \omega \sin \varphi V_{x}=\frac{2 \omega \cos \varphi}{a^{2} R} \int_{0}^{a} \Omega r^{3} d r=\frac{\Omega \omega \omega^{2}}{2 R} \cos \varphi .
\end{aligned}
$$

In oder to obtain an approximete feature of the motion of the vortex, we shall give a simple example.

Now, introducing $\Omega=\Omega_{0}+\omega\left(\sin \varphi_{0}-\sin \varphi\right)$, which is obtained from the law of conservation of absolute vorticity, into (25) and expanding with respect to $\varphi-\varphi_{0}$, we get

$$
\frac{\Omega \omega a^{2}}{2 R} \cos \varphi=\alpha+\beta y
$$

where

$$
\alpha=\frac{\Omega_{0} \omega c^{2}}{2 R} \cos \varphi_{0}, \quad \beta=-\frac{\omega^{2} a^{2}}{2 R^{2}}\left(\cos \varphi_{0}+\frac{\Omega_{0}}{\omega} \sin \varphi_{0}\right),
$$

ind the subscripts 0 mean initial values. Putting $2 \omega \sin \bar{\varphi}=: f$, where $\bar{\varphi}$ is the mean value of latitudes within the range of the motion, then (24) and (25) reduce to

$$
\frac{d^{2} x}{d t^{2}}-f \frac{d y}{d t}=0, \frac{d^{2} y}{d t}+f \frac{d x}{d t}=\alpha+\beta y .
$$

Solving these equations under the following initial conditions:

$$
t=0 ; x=0, y=0\left(\varphi=\varphi_{0}\right), V_{x}=V_{y}=0,
$$

we get

$$
\left\{\begin{array}{l}
x=\frac{a_{f} f}{f^{2}-\beta}\left(t-\frac{1}{\sqrt{f^{2}-\beta}} \sin \sqrt{f^{2}-\beta} t\right), \\
y=\frac{\alpha}{f^{2}-\beta}(1-\cos \gamma f-\bar{\beta} t) .
\end{array}\right.
$$

$\beta$ is negligibly small compared with $f^{2}$, because the ratio of them is $\beta / f^{2}=0\left(10^{-2}\right)$, 
then we get

$$
\left\{\begin{array}{l}
x=\frac{a}{f}\left(t-\frac{1}{f} \sin f t\right), \\
y=\frac{a}{f^{2}}(1-\cos f t) .
\end{array}\right.
$$

This is the equations of a cycloid, the centre of the circle moves on a line

$$
y=\frac{a}{f^{22}}=\frac{a^{2} \Omega_{0} \cos \varphi_{0}}{8 R \omega \sin ^{2} \varphi_{0}} \text { with a linear ve ocity } \frac{a}{f}=\frac{\Omega_{0} a^{2}}{4 R} \cot \varphi_{0}
$$

and the sense of rotation is anticyclonic. In Figs. 3 6, the radius ( $\mathrm{km}$ ), the velocity of the centre ( $\mathrm{km} /$ hour), the period (hour) and the trajectories are shown. In Fig. 3 and 4, the same curves are used for various radii by changing the scale of the ordinates.

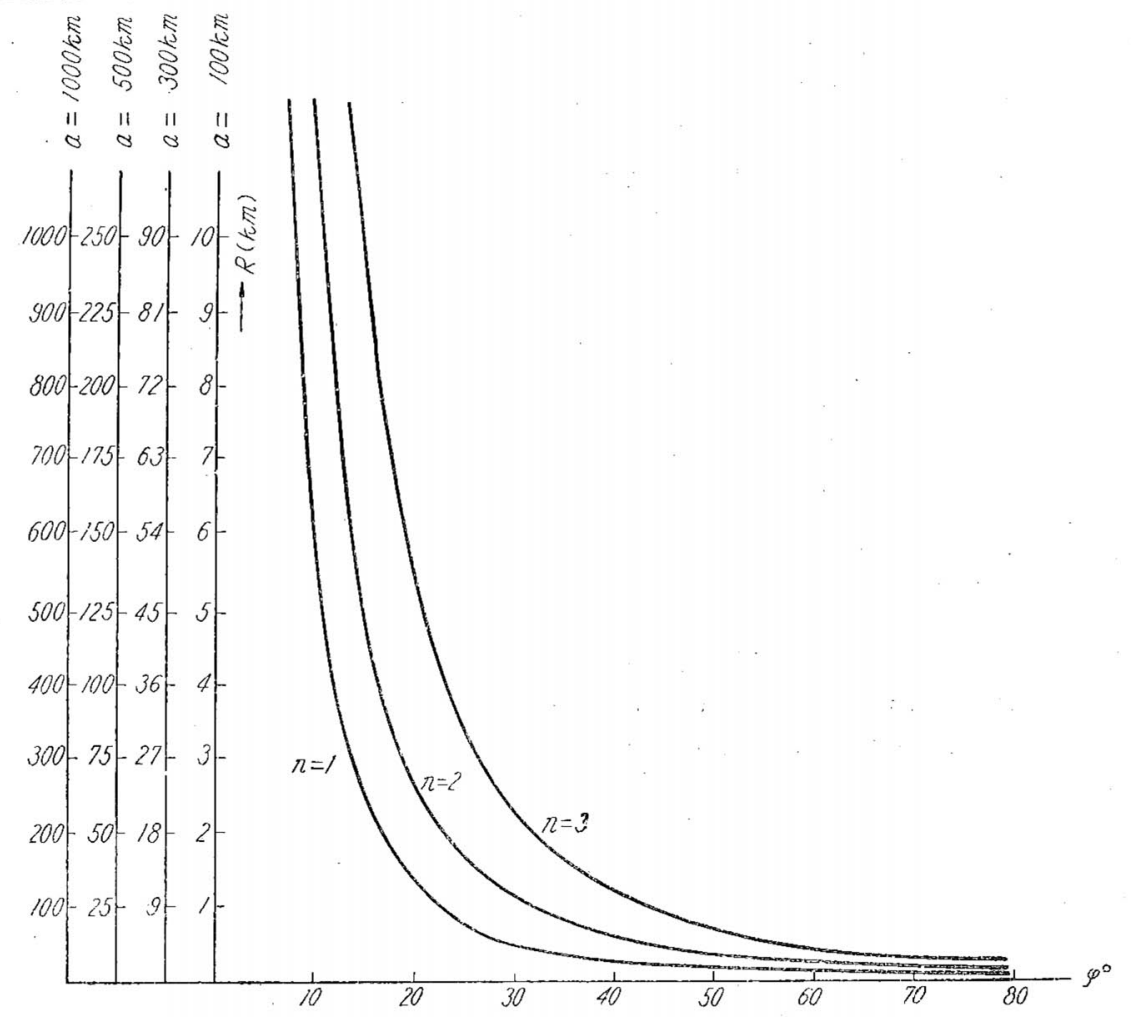

Fig. 3 Radii of the cycloid in $\mathrm{km}$.

Ordinate: the radii of the cycloid for various radii of vortices.

Ahscissa : the latitude of the center of vortices. $n: \frac{\Omega_{0}}{\omega}$

A cyclonic vortex moves eastward and an anticyclonic moves westward. From the above result, we can conclude that the combined effect of the Rossby's acceleration and the Coriolis acceleration cannot explain the meridional motion of vortices. The theory, rather, explains the meandering motion of tropical cyclones.

(ii) Motion in a non-uniform stationary field 


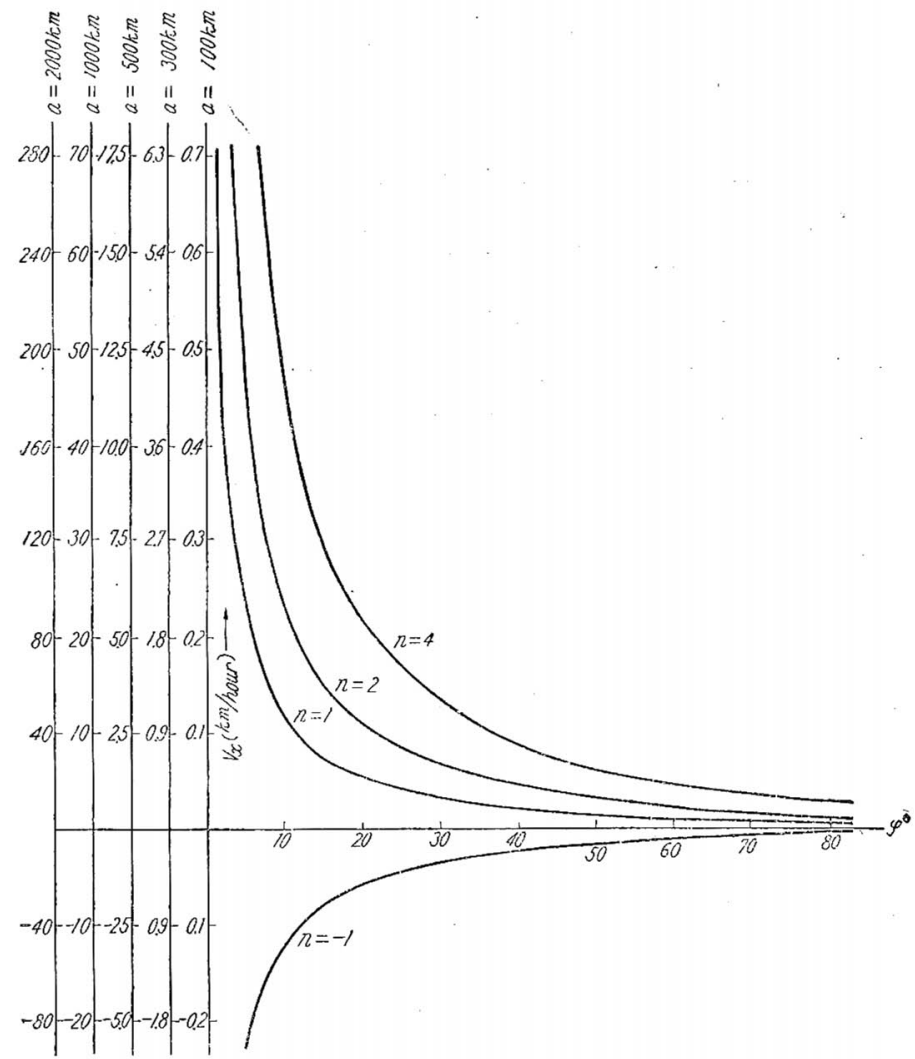

lig. 4 The eastward linear velocities in $\mathrm{km} / \mathrm{hour}$.

Ordinates: the linear velocities for various radii of vrotices. Abscissa: the latitudes of the centre of vortices. $n: \frac{\Omega_{0}}{\omega}$

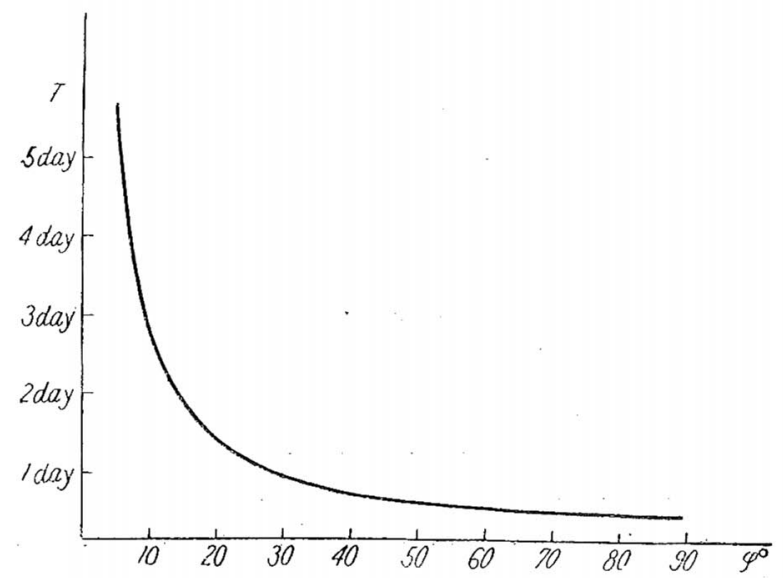

Fig. 5 Periods of revolution of the centre.

Ordinate: the period, Abscissa: the latitude of the centre of vortices 

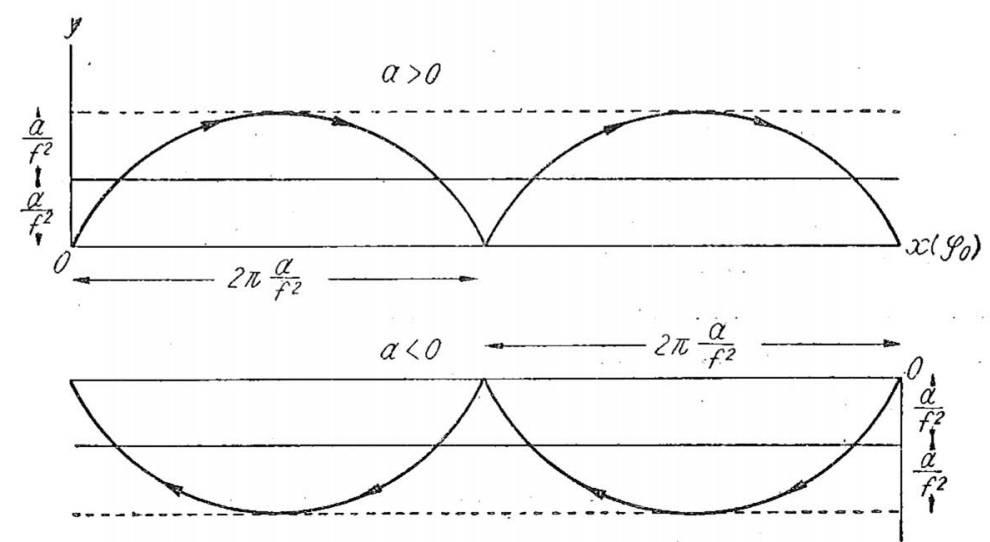

Fig. 6 Cycloidal path of vortices. $a>0$ :cyclonic vortices.

$$
a<0 \text { : anticyclonic vortices. }
$$

(a) The case of straight line isobars.

Now we consider the effect of the pressure gradient force upon the motion of a vortex.

If the isobars around the vortex $p^{\prime}$ are circular, the pressure gradient force due to $p^{\prime}$ does not give the effect upon the motion of the vortex. If the general pressure field $\bar{P}$ is a linear function of space coordinates, $\frac{I}{\rho} \frac{\partial \vec{P}}{\partial x}$ and $\frac{1}{\rho} \frac{\partial \bar{P}}{\partial y}$ are constant. Then the approximate equations of motion are given by

$$
\frac{d^{2} x}{d t^{2}}-f \frac{d y}{d t}=-\delta_{x}, \frac{d^{2} y}{d t^{2}}+f \frac{d x}{d t}=-\delta_{y}+\alpha+\beta y,
$$

where

$$
\delta_{x}=\frac{1}{\rho} \frac{\overline{\partial P}}{\partial x}, \delta_{y}=\frac{\mathrm{I}}{\rho} \frac{\overline{\partial P}}{\partial y} .
$$

Solving these equations under the following initial conditions:

$$
t=0 ; \quad x=0, y=0\left(\varphi=\varphi_{0}\right), \quad V_{x}=-\frac{\delta_{y}}{f}, \quad V_{y}=\frac{\delta x}{f},
$$

which mean that the vortex initially at $x=y=0$ is moving with the velocity of the geostrophic wind, we get

$$
\left\{\begin{array}{r}
x=\frac{\delta_{x} \beta}{f^{2}-\beta} \frac{t^{2}}{2}-\frac{\delta y}{f} t+\frac{\alpha f}{f^{2}-\beta}\left(t-\frac{1}{\sqrt{f^{2}-\beta}} \sin \sqrt{f^{2}-\beta} t\right) \\
-\frac{\delta_{x} \beta}{\left(f^{2}-\beta\right)^{2}}\left(1-\cos \sqrt{f^{2}-\beta} t\right) \\
y=\frac{\delta_{x}}{f \sqrt{f^{2}-\beta}} \sin \sqrt{f^{2}-\beta} t+\frac{\delta_{x} f}{f^{2}-\beta}\left(t-\frac{1}{\sqrt{f^{2}-\beta}} \sin \sqrt{f^{2}-\beta} t\right) \\
+\frac{a}{f^{2}-\beta}\left(1-\cos \sqrt{f^{2}-\beta} t\right) .
\end{array}\right.
$$

Since $\beta$ is small compared with $f^{2}$, we get

$$
\left\{x=\frac{\delta_{x} \beta}{f^{2}} \frac{t^{2}}{2} t-\frac{\delta_{y}}{f} t+\frac{a}{f}\left(t-\frac{1}{f} \sin f t\right)-\frac{\delta_{x} \beta}{f^{4}}(1-\cos f t),\right.
$$




$$
y=\frac{\delta_{x}}{f} t+\frac{a}{f^{2}}(1-\cos f t) .
$$

Comparing (35) with (31), we can see that the motion is composed of four types of motion (i) geostrophic motion (ii) cycioidal motion (iii) zonally accelerated motion (iv) oscillatory motion

(b) The case of parabolic isobars

Concerning the typhoon in the western Pacific, its motion along parabolic isobars is interesting, so that we shall study the problem.

The parabolic isobars are expressed by

$$
P=P_{A}+p_{1}\left(x-r_{A}\right)-p_{2}\left(y-y_{A}\right)^{2},
$$

where $x_{A}, y_{A}$ and $P_{A}$ are the coordinates and the pressure at the vertex of an isobar respectively. If the isobar passes through the origin, the condition

$$
p_{1} v_{A}+p_{2} y_{A}{ }^{2}=0
$$

holds. The pressure gradient forces are given by

then

$$
\frac{1}{\rho} \frac{\partial P}{\partial x}=\frac{\rho_{1}}{\rho}, \frac{I}{\rho} \frac{\partial P}{\partial y}=2 \frac{p_{2}}{\sigma} y_{A}-2 \frac{p_{2}}{\rho} y,
$$

$$
\delta_{x}=-\frac{p_{1}}{\rho}, \quad \delta_{y}=\frac{2 p_{3}}{\rho} y_{A}
$$

The equations of motion and initial conditions are as follows;

$$
\begin{aligned}
& \frac{d^{2} x}{d t^{2}}-f \frac{d y}{d t}=-\delta_{x}, \quad \frac{d^{2} y}{d t^{2}}+f \frac{d x}{d t}=\alpha-\delta_{y}+\left(\beta+\frac{2 p_{2}}{\rho}\right) y, \\
& t=0 ; \quad x=y=0, \quad V_{x}=-\frac{\delta_{y}}{f}=-\frac{2 p_{2}}{\rho f} y_{A} \quad V_{y}=\frac{\delta_{x}}{f}=\frac{p_{1}}{\rho f} .
\end{aligned}
$$

The solutions are readily written down as follows;

$$
\begin{aligned}
x= & \frac{p_{1}}{\rho} \frac{\left(\beta+\frac{2 p_{2}}{\rho}\right)}{\left(f^{2}-\beta-\frac{2 p_{2}}{\rho}\right)} \frac{t^{2}}{2}-\frac{2 p_{2}}{\beta f} y_{A} t+\frac{\alpha f}{f^{2}-\beta-\frac{2 p_{2}}{f}}\left(t-\frac{1}{\sqrt{f^{2}-\beta-\frac{2 p_{2}}{f}}} \times\right. \\
& \sin \sqrt{\left.f^{2}-\beta-\frac{2 p_{2}}{f} t\right)}-\frac{p_{1}}{\rho} \frac{\left(\beta+\frac{2 p_{3}}{\rho}\right)}{\left(f^{2}-\beta-\frac{2 p_{2}}{\beta}\right)}\left(1-\cos \sqrt{f^{2}-\beta-\frac{2}{\rho} p_{2}} t\right), \\
y= & \frac{p_{1}}{\rho f}-\frac{1}{\sqrt{f^{2}-\beta-\frac{2}{\rho} p_{2}}} \sin \sqrt{f^{2}-\beta-\frac{2 p_{9}}{\rho} t} \\
+ & \frac{p_{1} f}{\rho\left(f^{2}-\beta-\frac{2 p_{3}}{\rho}\right)}\left(t-\frac{1}{\sqrt{f^{2}-\beta-\frac{2 p_{2}}{f}}} \sin \sqrt{\left.f^{2}-\beta-\frac{2 p_{2}}{\rho} t\right)}\right. \\
& +\frac{\alpha}{f^{2}-\beta-\frac{2 p_{2}}{f}}\left(1-\cos \sqrt{f^{2}-\beta-\frac{2 p_{2}}{f}} t\right),
\end{aligned}
$$

Neglecting $\beta$ compared with $f^{2}$, we get 


$$
\begin{aligned}
& x=\frac{p_{1}}{\rho} \frac{\beta}{\left(f^{\prime \prime}-\frac{2 p_{2}}{\rho}\right)} \frac{t^{2}}{2}+\cdots-\frac{p_{1} p_{3} t^{2}}{\rho^{2}\left(f^{\prime}-\frac{2 p_{3}}{\rho}\right)}-\frac{2 p_{3}}{\rho_{t} y_{A}} y_{A}+\frac{\alpha f}{f^{2}-\frac{2 p_{3}}{\rho}}\left(t-\frac{1}{\sqrt{f^{2}-\frac{2 p_{3}}{\rho}}} \times\right.
\end{aligned}
$$

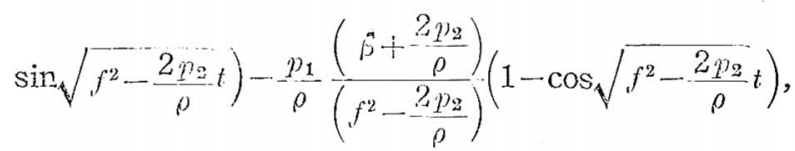

$$
\begin{aligned}
& y=\frac{p_{1}}{\rho f \sqrt{f^{2}-\frac{2 p_{3}}{\rho}}} \sin \sqrt{f^{2}-\frac{2 p_{9}}{\rho}} t+\frac{p_{1_{2}}}{\rho\left(f^{2}-\frac{\left.2 p_{2}\right)}{\rho}\right)}\left(t-\frac{1}{\sqrt{f^{2}-\frac{2 p_{3}}{\rho}}} \sin \sqrt{f^{2}-\frac{2 p_{2}}{\rho}} t\right) \\
& +\frac{\alpha}{f^{2}-\frac{2 p_{2}}{\rho}}\left(1-\cos \sqrt{f^{2}-\frac{2 p_{3}}{\rho} t}\right) \text {. }
\end{aligned}
$$

The motion is composed of four types of motion (i) quasi-gradient wind motion approximately along isobars (ii) cycloidal motion (iii) zonally accelerated motion (iv) oscillatory motion.

Now we consider the quasi-gradient wind motion along isobars.

The equations

$$
x=\frac{p_{1} p_{2} t^{2}}{v^{2}\left(f^{2}-\frac{2 p_{2}}{\rho}\right)}-\frac{2 p_{2} y_{A}}{\rho f^{\prime}} t, \quad y=\frac{p_{1} f^{\prime}}{\rho\left(f^{2}-\frac{2 p_{2}}{\rho}\right)} t
$$

are the parametric representation of a parabota

$$
x-\left(1-\frac{2 p_{2}}{\rho f^{2}}\right) \cdot x_{A}=\frac{p_{1}}{\rho}\left(1-\frac{2 p_{2}}{\rho f^{2}}\right)\left(y-y_{A}\right)^{2} .
$$

This parabola passes through the origin, but it is elongated zonally compared with the original isobar. The westward deviation at the vertex from that of the isobar is $\frac{2 p_{2}}{\rho f_{2}^{2}} x_{A}$. If the coordinates of the vertex of the isobar passing through the origin $\left(140^{\circ} \mathrm{E}, 10^{\circ} \mathrm{N}\right)$ are $\left(130^{\circ} \mathrm{E}, 25^{\circ} \mathrm{N}\right)$ and the meridional distance at the origin between the isobar and the next isobar, meridional distance at the initial isobar, $2 \mathrm{mb}$ lower than the former, is $1000 \mathrm{~km}$, we get $x_{A}=1000 \mathrm{~km}, \frac{2 p_{9}}{\rho}=(2 / 1.5) \times 10^{-10}$ c.g.s. Putting $f=4 \cdot 2 \cdot 10^{-5} \mathrm{sec}^{-1}$, the value at $\varphi=18^{\circ} \mathrm{N}$ and $x_{A}=1000 \mathrm{~km}$, the deviation becomes $75 \mathrm{~km}$. Then the trajectory is approximately equal to the parabolic isobax.

Now we consider the basic current,

$$
\overline{V_{x}}=-\frac{I}{\rho} \frac{\partial \bar{P}}{\partial y}=-\frac{p_{9}}{\rho f} y_{11}+2 \frac{p_{2}}{\rho f} y .
$$

Then $\frac{\partial \bar{V}_{x}}{\partial y}=2 \frac{p_{2}}{\rho f}$. The positive $\frac{\partial \vec{V}_{x}}{\partial y}$ means anticyclonic shear. Then the anticyclonic shear acts so as to increase the period of the oscillatory motion. If $f^{\prime \prime}<\frac{2 p_{2}}{\rho}$ the oscillatory motion becomes unstabie. This resuit coinsides with the criterion for stability by the parcel method. But this case does not occur in the atmosphere. If we take above value of $\frac{2 p_{2}}{\rho}$ and $f$ at $\bar{\varphi}=18^{\circ} \mathrm{N}$ i. e. $4 \cdot 2 \cdot 10^{-5} \mathrm{sec}^{-1}, \frac{2 p_{2}}{\rho}$ is smaller than 
$f^{\prime \prime}$, but is not negligible.

Now we shall give a rough estimation of the zonal deviation from the isobar due to the non-periodic terms. We assume

$$
\alpha=10^{-2}, f=4 \cdot 10^{-5}, \frac{\alpha f}{f^{2}-\frac{2 p_{2}}{\rho}}=1 / 4 \cdot 10^{3},
$$

then the deviation of a cyclone from the isobar during 1 hour is $10 \mathrm{~km}$ eastward. Further we assume $\beta=-2 \cdot 10^{-11}, \rho=10^{-3}, f=4 \cdot 10^{-5}, p_{1}=2 \cdot 10^{-5}$, then $\frac{p_{1} \beta}{\rho f^{2}} \frac{1}{2}=20 \mathrm{~m} /$ hour ${ }^{2}$, then if $t=100$ hour, the westward deviation due to this term attains $200 \mathrm{~km}$.

(iii) Nonstationary pressure field

Next we consider the case, where the pressure varies with time. The equations of motion become

$$
\begin{aligned}
& \frac{d V_{x}}{d t}-2 \omega \sin \varphi V_{y}=-\frac{1}{\rho} \frac{\overline{\partial P}}{\partial x}+G_{x} \\
& \frac{d V_{y}}{d t}+2 \omega \sin \varphi V_{x}=-\frac{1}{\rho} \frac{\partial \bar{P}}{\partial y}+G_{y}
\end{aligned}
$$

Differentiating with respect to $t$, we get

$$
\begin{aligned}
& \frac{d^{2} V_{x}}{d t^{2}}-2 \omega \sin \varphi \frac{d V_{y}}{d t}-\frac{2 \omega \cos \varphi}{R} V_{y}=\frac{d}{d t}\left(-\frac{1}{\rho} \frac{\partial \bar{P}}{\partial x}\right)+\frac{d G_{x}}{d t} \\
& \frac{d^{2} V_{y}}{d t^{2}}+2 \omega \sin \varphi \frac{d V_{x}}{d t}+\frac{2 \omega \cos \varphi}{R} V_{x}=\frac{d}{d t}\left(-\frac{1}{\rho} \frac{\partial \bar{P}}{\partial y}\right)+\frac{d G_{y}}{d t} .
\end{aligned}
$$

Then we get

$$
\begin{aligned}
& \frac{d V_{x}}{d t}=-\frac{1}{f} \frac{d^{2} V_{y}}{d t^{2}}-\frac{1}{f} \frac{d}{d t}\left(\frac{1}{\rho} \frac{\partial \bar{P}}{\partial y}\right)+\frac{1}{f} \frac{d G_{y}}{d t}-\frac{1}{R} \cot \varphi V_{x}, \\
& \frac{d V_{x}}{d t}=+\frac{1}{f} \frac{d^{2} V_{y}}{d t^{2}}+\frac{1}{f} \frac{d}{d t}\left(\frac{1}{\rho} \frac{\partial \bar{P}}{\partial x}\right)-\frac{1}{f} \frac{d G_{x}}{d t}-\frac{1}{R} \cot \varphi V_{y} .
\end{aligned}
$$

Introducing (52) and (53) into (48) and (49) and rearranging, we get

$$
\begin{aligned}
& V_{x}=-\frac{1}{\rho f} \frac{\partial \vec{P}}{\partial x}+\frac{1}{f} G_{y}-\frac{1}{f^{2}} \frac{d^{2} V_{x}}{d t^{2}}-\frac{1}{f^{2}} \frac{d}{d t}\left(\frac{1}{\rho} \frac{\partial \bar{P}}{\partial x}\right)+\frac{1}{f^{2}} \frac{d G_{x}}{d t}, \\
& V_{y}=+\frac{1}{\rho f} \frac{\partial \bar{P}}{\partial y}-\frac{1}{f^{\prime}} G_{x}-\frac{1}{f^{2}} \frac{d^{2} V_{y}}{d t^{2}}-\frac{1}{f^{2}} \frac{d}{d t}\left(\frac{1}{\rho} \frac{\partial \bar{P}}{\partial y}\right)+\frac{1}{f^{2}} \frac{d G_{y}}{d t} .
\end{aligned}
$$

Here $\frac{1}{R} \cot \varphi V_{x}$ and $\frac{1}{R} \cot \varphi V_{y}$ are neglected compared with $f V_{x}$ and $f V_{y}$.

$$
\begin{aligned}
& -\frac{1}{f^{2}} \frac{d}{d t}\left(\frac{1}{\rho} \frac{\partial \bar{P}}{\partial x}\right),-\frac{1}{f^{2}} \frac{d}{d t}\left(\frac{1}{\rho} \frac{\partial \bar{P}}{\partial y}\right), \\
& -\frac{1}{f^{2}} \frac{d}{d t}\left\{\frac{1}{\rho a} c_{1}(\alpha)\right\},-\frac{1}{f^{2}} \frac{d}{d t}\left\{\frac{1}{\rho a} \widetilde{c_{1}(\alpha)}\right\}
\end{aligned}
$$

mean isallobaric velocity components. Hence it is to be noticed the isallobaric gradient is taken referred to the vortex. If we use the ordinary isallobars, we must transform with the formula $\frac{d}{d t}=\frac{\partial}{\partial t}+V_{x}-\frac{\partial}{\partial x}+V_{y} \frac{\partial}{\partial y}$.

Then we get 


$$
\begin{aligned}
V_{x}=-\frac{1}{\rho f} \frac{\partial \bar{P}}{\partial x} & +\frac{1}{f} G_{y}-\frac{1}{f^{2}} \frac{d^{2} V_{x}}{d t^{2}}-\frac{1}{f^{2} \rho}\left(\frac{\partial \bar{P}}{\partial x}+V_{x} \frac{\partial}{\partial x}\left(\frac{\partial \bar{P}}{\partial x}\right)\right. \\
& \left.+V_{y} \frac{\partial}{\partial y}\left(\frac{\partial \bar{P}}{\partial x}\right)\right)+\frac{1}{f^{2}} \frac{d G_{x}}{d t}, \\
V_{y}=+\frac{1}{\rho f} \frac{\partial \bar{P}}{\partial x} & -\frac{1}{f} G_{x}-\frac{1}{f^{2}} \frac{d^{2} V_{y}}{d t^{2}}-\frac{1}{f^{2} \rho}\left(\frac{\partial \bar{P}}{\partial y}+V_{x} \frac{\partial}{\partial x}\left(\frac{\partial \bar{P}}{\partial y}\right)\right. \\
& \left.+V_{y} \frac{\partial}{\partial y}\left(\frac{\partial \bar{P}}{\partial y}\right)\right)+\frac{1}{f^{2}} \frac{d G_{y}}{d t},
\end{aligned}
$$

where the dotts denote the timely variations referred to the earth.

For the practical purpose, we can neglect the second derivatives of the space coordinates and the timely change of the acceleration. Then amalgamating $\frac{1}{a} c_{1}(a)$ and $\frac{\mathrm{I}}{a} \widetilde{c_{1}}(a)$ and there timely derivatives into $\bar{P}$ and its timely derivative respectively, we get

$$
\begin{aligned}
& V_{x}=-\frac{1}{\rho \dot{f}^{\prime}} \frac{\partial \bar{P}}{\partial x}-\frac{1}{\rho f^{2}} \frac{\partial \overline{\dot{P}}}{\partial \mathrm{x}}+\frac{\mathrm{I}}{a^{2} R} \cot \varphi_{0} \int_{0}^{a} b_{0}(r) r^{2} d r-\frac{\cos \varphi_{0}}{2 a^{2} R \omega \sin 2 \varphi_{0}} \int_{0}^{a} a_{0}(r) r^{2} d t \\
& V_{y}=+\frac{1}{\rho f} \frac{\partial \bar{P}}{\partial y}-\frac{1}{\rho f^{2}} \frac{\partial \overline{\dot{P}}}{\partial y}-\frac{\mathrm{I}}{a^{2} R} \cot \varphi_{0} \int_{0}^{a} a_{0}(r) r^{2} d r-\frac{1}{2 a^{2} R \omega} \frac{\cos \varphi_{0}}{\sin ^{2} \varphi_{0}} \int_{0}^{a} b_{0}(r) r^{2} d r
\end{aligned}
$$

The first and second terms or these equations have the same form as the geostrophic wind and the isallobaric wind, provided that the mean values of pressure and their timely variations are used. Further we can amalgamate integral term into $P$ and $\dot{P}$, if we use the pressure-equivalent of them. From (58) and (59) we conclude that vortices move in the direction of a vector composed of the geostrophic velocity and the isallobaric velocity.

\section{Remark on the metric accelerations}

In the above investigations the metric accelerations $-\frac{V_{x} V_{y}}{R} \operatorname{tg} \varphi$ and $\frac{V x^{2}}{R} \operatorname{tg} \varphi$ are neglected in (3) and (4). If we apply formal operation mentioned in 3 , we get

$$
\begin{aligned}
& \frac{1}{S} \iint_{S}\left(-\frac{V_{x} V_{y}}{R} \operatorname{tg} \varphi\right) d S=-\operatorname{tg} \varphi_{0} \frac{V_{x} V_{y}}{R}-H_{1} V_{x}-F_{2} V_{y}-H_{x} \\
& \frac{1}{S} \iint_{S} \frac{V_{x}^{2}}{R} \operatorname{tg} \varphi d S=\frac{V_{x}^{2}}{R} \operatorname{tg} \varphi_{0}+2 F_{2} V_{x}-H_{y}
\end{aligned}
$$

where

$$
\begin{aligned}
& F_{1}=\frac{1}{R^{2} a^{2} \cos ^{2} \varphi_{0}} \int_{0}^{a} a_{0}(r) r^{2} d r, \\
& F_{\Xi}=-\frac{1}{R^{2} a_{t}^{2} \cos ^{2} \varphi_{0}} \int_{0}^{a} b_{0}(r) r^{2} d r, \\
& H_{x}=\frac{2 \omega}{a^{2} R} \cos \varphi_{0} \int_{0}^{a} a_{0}(r) r^{2} d r, \\
& H_{y}=\frac{2 \omega}{a^{2} R} \cos \varphi_{0} \int_{0}^{a} b_{0}(r) r^{2} d r-\frac{1}{a^{2} R} t g \varphi_{0} \int_{0}^{a}\left\{a_{0}^{2}(r)+b_{0}^{2}(r)\right\} r d r_{0}
\end{aligned}
$$


The effect of $F_{1}$ and $F_{2}$ are negligible compared with the Rossby's acceleration. $H_{x}$ and $H_{y}$ are, however, comparable of magnitude with the Rossby's acceleration. and is rather predominant than the latter in high latitudes. But since, at present, there is certain questionable point in the physical meanings of $H_{x}$ and $H_{y}$, we have put them out of account in the above investigation. The detailed discussions of them are laid for future investigation.

\section{References}

Bjerknes, J. and Holnboe, J. 1944 : On the theory of cyclones. J. Meteor., $\mathbf{1}, 1-22$.

Kuo, H. L. 1950: The atmospheric vortices and the general circulation., J. Meteor. 7, 247 $-258$

Rossby, C. G. 1948: On the displacement and intensity changes of atmospheric vortices. J. Marine Res. 5, 175-196.

1949: On the mechanism for the release of potential energy in the atmosphere. J. - Meteor., 6, 163-180.

Yeh, T. C. 1950: The motion of tropical storns under the influence of a superposed southerly current. J. Meteor., 7, 108-113. 